\title{
Oral Surgery in and after the COVID-19 Period from the Consultation to the Operating Room
}

\author{
Asma Azzouzi, Saliha Chbicheb (iD \\ Department of Oral Surgery, Dental Consultation and Treatment Center, Ibn Sina University Hospital Center, Faculty of \\ Dental Medicine, Mohammed V University, Rabat, Morocco
}

\begin{abstract}
Severe acute respiratory syndrome (SARS) caused by the SARS-CoV-2 virus was first reported in Wuhan, China. In December 2019 has rapidly spread worldwide at an unprecedented rate and has profound health and socioeconomic effects directly affecting all fields. From the onset of the pandemic, the authors realized the impending impact on the dental field. Dental professionals are uniquely exposed to environments with high levels of infection risk from aerosols and oral fluids. Dental treatment has become a challenge during the epidemic. However, the light of optimism has faded with the onset of vaccination. The purpose of this article is to guide dental surgeons on how to adapt their current practice during and after the COVID-19 pandemic concerning consultation activity, surgical activity, and drug prescribing in oral surgery. Thus, an electronic search was performed from PubMed (MEDLINE), Scopus, Cochrane, Google Scholar, and ScienceDirect databases. Thirty-five articles were identified, of which 24 were selected to compose this article. Within the limitations of this literature review, the presence of the virus is inevitable in oral surgery practice. Dentists must continue to provide care to their patients while monitoring the evolution of Covid-19.
\end{abstract}

KEYWORDS: COVID-19, SARS-CoV-2, Coronavirus, oral surgery, oral Infections, Oral bleeding, medical prescriptions.

Correspondence: Asma Azzouzi, Department of Oral Surgery, Dental Consultation and Treatment Center, Ibn Sina University Hospital Center, Rabat, Morocco. Email: asmaazzouzi1993@gmail.com

Copyright (C) 2021 Azzouzi A \& Chbicheb S . This is an open access article distributed under the Creative Commons Attribution 4.0 International, which permits unrestricted use, distribution, and reproduction in any medium, provided the original work is properly cited.

\section{INTRODUCTION}

On January 30, 2020, the World Health Organization (WHO) announced that the epidemic caused by the SARS-CoV-2 virus had constituted a public health emergency of international concern (1). This epidemic had had a significant impact on the management of patients in all surgical and medical specialties. Clinical practices must be adapted to protect caregivers and patients. (2)

SARS-CoV-2 resides primarily within the respiratory tract, with high viral loads in the oral and nasal cavities. (3) Dentistry has certain specificities that make it a unique medical discipline because of the -exposure of the practitioner, the assistant, and the patients to the risk of contamination. Indeed, the proximity between practitioner and patient (less than $35 \mathrm{~cm}$ during care), the invasive care delivered to patients with exposure to saliva, blood, and other fluids, and the -use of complex instrumentation generating aerosols make the risk of contamination in the dental office real (4). Thus, any diagnostic or therapeutic procedure at the level of the oral cavity involves a risk of dissemination of the virus and contamination of the care team by inhalation of contaminated droplets or ocular projections or by indirect transmission by hand or via contaminated surfaces or objects (5)

\section{MATERIALS AND METHODS}

A comprehensive literature search was performed in Pubmed (MEDLINE), Scopus, Cochrane, Science Direct, and google scholar databases, published in 2020-2021. The following keywords were COVID-19, SARS-CoV-2, Coronavirus, oral surgery, oral infections, oral bleeding, medical prescriptions. Articles were selected on the basis of title, abstract, material, and methods. The inclusion criteria were literature reviews, surveys. However, the only exclusion criterion was the publication of studies before 2020 . 


\section{RESULTS AND DISCUSSIONS}

In oral surgery and other dental specialties, medical and paramedical personnel are particularly vulnerable to contamination. Dentists, dental hygienists, dental assistants, and receptionists must keep their infection control knowledge and skills current and follow protocols recommended by the appropriate authorities to protect themselves and their patients from infection.

To reduce the risk of SARS CoV-2 infection, given the current health crisis and the way the disease is spreading, an attempt should be made to telephone triage all patients who require dental care (6) to assess whether:

The patient has symptoms of SARS CoV-2 infection, including:

-fever $\left(\mathrm{T}^{\circ}>\right.$ to $\left.38^{\circ} \mathrm{C}\right)$ or feeling of fever;

-cough;

-chest pain with coughing;

-dyspnea;

-digestive signs;

-headache;

-anosmia \pm agueusia of less than one month;

The patient has been in contact with COVID-19 positive persons for at least $15 \mathrm{~min}$.

In oral surgery, the following three modes of telecommunication are of great value for oral diagnosis. (8)

- Tele-referral allows professionals to screen, guide and refer patients as needed.

Remote monitoring allows professionals to visually monitor suspected or positive SARS-CoV-2 patients with oral lesions through photographic control.

Finally, teleconsultation allows for exchanging information between professionals, helping to diagnose and initiate therapy with the patient with greater agility and precision.

A study published by Petruzzi et al. (7) confirmed the use of WhatsApp as a tool in oral diagnosis, in which $82 \%$ of the tele-consulting cases agreed with the clinicopathological diagnosis, suggesting that it is a good option for teledentistry.

Thus, the questionnaire should be used in its entirety at the time of the patient's call and at the time of the patient's visit to learn about the patient's care needs and identify possible risk situations. The dentist must collect accurate information about the patient's health status to prescribe appropriate medications and provide detailed instructions for safe home care in cases where dental treatment may be delayed.

Who to see in the consultation?

After teleconsultation, only urgent consultations are to be seen clinically. In oral surgery, it is any situation that requires intervention within 24 hours to avoid serious complications, among the most frequent cases:

- Infections of the oral cavity accompanied by swelling and general symptoms (lymph nodes, fever, malaise)

- Oral bleeding (including post-operative bleeding)

\section{Tumefaction / oral infection}

Clinical elements to look for

- Age

- Health problems, ongoing treatments, or allergies, you should look specifically for :

An immunosuppressed background (treatment or pathology)

Self-medication with NSAIDs
Altered general condition (fever, asthenia)

- Date of onset of symptoms

- Location of swelling: endo or exo-buccal: Exclude periodontal abscess versus maxillofacial cellulitis

- Skin changes: erythema, heat, crepitation on palpation, fistula

- Pain on percussion or palpation of a tooth

Severity criteria

- Associated trismus

- Submandibular or sublingual swelling with difficulty or pain in swallowing

- Swelling that spreads to the eye: swelling of the eyelids, difficulty opening or closing the eye

- Erythema or swelling that extends into the neck

- Crepitation on palpation of the swelling

- Fever and asthenia

Orientation

$\varnothing$ Patients without urgent consultation needs

- Clinical signs in favor of a simple periodontal abscess or a vestibular voussure.

Lack of severity criteria.

- Avoid the causal tooth.

- Prescription of analgesic and antiseptic mouthwash.

- Recall of the patient at $48 \mathrm{~h}$, if no improvement: 1 st line antibiotic prescription.

- Recall of the patient at $48 \mathrm{~h}$, if no improvement:

Consultation of the dental surgeon on duty

- Localized endo/exobuccal swelling without signs of severity or only trismus in a patient without

immunosuppression factors.

- First-line antibiotic prescription.

- Recall the patient at 48 h, if no improvement and still no criteria of severity: 2 nd line antibiotic prescription.

- Recall of the patient at $48 \mathrm{~h}$, if no improvement:

Consultation of the dental surgeon on duty

$\varnothing$ Patients requiring an urgent consultation with the

on-call dental surgeon

- Endo/exobuccal swelling in a pregnant woman or a frail patient

- Localized swelling without signs of severity in a patient with immunosuppression

- First-line antibiotic prescription while waiting for an appointment with the on-call dental surgeon

$\varnothing$ Patients requiring urgent consultation in a hospital emergency department

- Swelling with severity criteria

Oral hemorrhage

Clinical elements to look for

- Age

- Health problems, current treatments or allergies, you should look specifically for :

- Taking anticoagulants or antiplatelets. For patients on VKA, ask for the last INR result

- Risk factors (thrombocytopenia, thrombopathy, hemophilia, Willebrand's disease, severe hepatic disorders)

- Presence of bruises or purple spots elsewhere on the body

- The location of the bleeding

- Estimation of the amount of blood

- Cause of bleeding: shock, extraction, bite or spontaneous

- Date of onset of bleeding

\section{Severity criteria}

- Elderly patient alone or dependent who may not follow advice given 
- Bleeding that persists after compression for more than 15 minutes (1)

- Patients on anticoagulants or with pathologies at risk of bleeding

\section{Orientation}

$\diamond \quad$ Patients without urgent consultation needs

After 15 minutes of compression, the bleeding stopped in a patient without treatment, without medical history, with an identified cause.

$\diamond \quad$ Patients requiring an urgent consultation with the on-call dental surgeon

No history of bleeding but bleeding persists after 15 minutes of compression

$\diamond \quad$ Patients requiring urgent consultation in a hospital ward (2)

Persistent bleeding in a patient with a bleeding risk related to his treatment or pathology.

\section{Medical advice and prescriptions}

- Do not spit, do not use mouthwash, apply ice to the exoand endobuccal area of the bleeding. If the bleeding resumes, repeat the 15-minute compression.

Do not take NSAIDs or corticosteroids.

For a patient on anticoagulants: Compress the bleeding site with tranexamic acid-soaked compresses folded in four and reassess 15 minutes later. Depending on the clinical situation, it is possible to make a prescription by phone.

\section{Dental surgeon in the operating room}

Oral care in oral surgery can be classified into:

- Aerosolized Care: Many devices used in oral surgery can generate aerosols that are sources of contamination for patients and caregivers. Ex: Complex extraction/wisdom teeth Apical, cystic, tumor, and implant surgery.

- Non-aerosolizing care: these are procedures that do not generate sprays, such as simple extraction without rotating equipment and suture removal

When performing operations on a patient of unknown COVID-19+ or COVID-19 status, the room's furniture and equipment should be kept to a minimum, and only those essential to the surgical procedure should be present. Spaces should always be closed, and entry/exit and the number of nursing staff should be kept to a minimum. (14) Hand hygiene is a standard measure in dental practice but is of increasing importance in limiting the transmission of SARS-CoV-2. Lotfinejad et al. (2020) (12) showed the effectiveness of alcohol-based solutions on inactivated enveloped viruses, including coronaviruses, suggesting the use of solutions containing at least $60 \%$ ethanol for hand hygiene. (13)

During all aerosolizing / non-aerosolizing treatments, the practitioner and his assistant wear a protective gown/cap. Wear an FFP2 mask (or equivalent, e.g., N95, KN95, etc.) during aerosol-generating care without a valve. In the context of risk of mask shortage, the FFP2 mask can be kept during a care shift (4h) (extended use) involving several treatments/patients, provided that it has not been directly soiled, wet, or handled. Its recycling or reuse cannot be envisaged at present. The process of autoclaving at $134^{\circ} \mathrm{C} / 18$ minutes, under saturated steam pressure, does not seem suitable and deteriorates the functional filtration properties of the masks (16)

For non-aerosolized procedures, a surgical mask is recommended. However, air exhaled by the wearer through the valve may contaminate the outside environment.
- Charlotte (or cap) is mandatory: it can be kept between each patient for half a day if it is not soiled or wet. It can be single-use or washable; Protective glasses and visors with lateral bypass allow to protect the eyes and the mask from any soiling or direct contamination. They must be disinfected after each patient. (19)

- On the other hand, wearing overshoes is not justified because of the risk of contamination when handling them during installation and removal.

- Single-use gloves;

Have an antiseptic mouthwash performed

$1 \%$ Hydrogen peroxide

$0.2 \%$ Povidone-iodine

$0.2 \%$ Chlorhexidine

$2 \%$ Listerine

The patient should hold the solution in the mouth for 1 minute while avoiding using a spittoon. Have the patient aspirate the mouthwash after gargling. $(9,10,11,14)$

The dentistry practice involves rotating dental/surgical instruments, which create a high volume of aerosols that may contain a mixture of water, saliva, blood, microorganisms, and other debris. (14-20). If the use of a rotating aerosol-creating instrument is necessary and unavoidable: Use a red or blue-ringed contra-angle handpiece instead of a turbine; Adjust the water flow rate to the minimum necessary, otherwise minimal noncontinuous use of the handpiece at high speed for short periods is not considered to be at risk of producing aerosols from body fluids.

In case of suture, preferably use an absorbable suture.

\section{Covid 19 and medical prescriptions in oral surgery}

Despite the decrease in the total number of prescriptions during the lock-in period, reflecting a reduction in the total number of dental visits, the use of antibiotics and opioid analgesics during this period remained relatively high, consistent with increased use of these drugs as an alternative to routine surgical treatment to reduce the transmission and spread of COVID-19. (17) Furthermore, COVID-19 pharmacotherapy is likely to impact dental practice because a significant number of these pharmacotherapeutic agents are widely used for oral surgical patients, including analgesics and antibiotics. Non-opioid analgesics, namely paracetamol, and NSAIDs are recommended for COVID-19 and dental disease for their antipyretic and analgesic effects. Azithromycin is also a preferred antibiotic in the dental setting for specific patient categories. (15-21)

Following the relaxation of lockout restrictions, prescriptions for broad-spectrum antibiotics and opioid analgesics have increased, reflecting clinical deterioration due to delayed treatment during the lockout period. (17)

Dental practitioners should keep in mind that corticosteroids may be considered in some treatment protocols in COVID-19 patients who may subsequently develop side effects. (15) One study (Le Bon, 2021) showed that combining a short course of oral corticosteroids and olfactory training may be beneficial in helping patients with persistent dysosmia recover from olfactory loss due to COVID-19. (18)

Other COVID-19 drugs such as antivirals and hydroxychloroquine are less relevant to dental practice, and their association with long-term adverse effects requires sufficient awareness among dental practitioners. 
Finally, the choice of appropriate drug therapy for the oral surgery patient involves the therapeutic indications, potential adverse effects, and the individual systemic status of the patient. (15-21).

\section{Oral surgery after the COVID-19 Pandemic}

The return to regular oral surgery practice, especially after the COVID-19 vaccination, meant great relief and reduced anxiety for health care personnel and a resumption of patient flow as before the COVID-19 epidemic.

However, the study by Salgarello S et al. (2021) highlighted that the return to normalcy has a different meaning after one year of the COVID-19 outbreak. Job insecurity and fear of COVID-19 continue to characterize daily dental activity just as in the early days of the COVID-19 pandemic. (22)

Therefore, the full recovery of dental activity after the COVID-19 pandemic has imposed a new concept of safety

\section{AUTHORS' CONTRIBUTIONS}

All the authors have actively participated in the redaction, the revision of the manuscript, and provided approval for this final revised version.

\section{REFERENCES}

[1] Meng L, Hua F, Bian Z. Coronavirus Disease 2019 (COVID-19): Emerging and Future Challenges for Dental and Oral Medicine. J Dent Res. 2020 May;99(5):481-487. DOI : $\underline{10.1177 / 0022034520914246}$

[2] Radulesco T, Verillaud B, Béquignon E, Papon J F, Jankowski R, De Gabory LLT, et al. COVID-19 et rhinologie: conseils de bonne pratique, de la consultation au bloc opératoire. Ann fr d'Oto-rhino-laryngologie Pathol Cervico-faciale. $2020 \quad$ Sept;137(4): 286-291. DOI: $10.1016 \% 2$ Fj.aforl.2020.04.010

[3] Zou L, Ruan F, Huang M, Liang L, Huang H, Hong Z, et al. SARS-CoV-2 viral load in upper respiratory specimens of infected patients. N Engl J Med. 2020 Mar 19;382(12):1177-9. DOI: $10.1056 /$ nejmc2001737

[4] Yahya IB, Bentahar Z, Chlyah A, Haitami S, El bouhairi M. Guide à l'usage des médecins dentistes du maroc. Covid-19 : mesures de prévention de la contamination en pratique dentaire. African J Denti Implantol. 2020; no 1. [Accessed 2021 Nov 29]. Available From: https://revues.imist.ma/index.php/AJDI/article/view/2444 5

[5] Givi B, Schiff BA, Chinn SB, Clayburgh D, Iyer NG, Jalisi $S$, et al. Safety recommendations for evaluation andsurgery of the head and neck during the COVID-19 pandemic. JAMA Otolaryngol Head Neck Surg. 2020 Jun 1; 146(6):579-584. DOI: $\underline{10.1001 / j a m a o t o .2020 .0780}$

[6] Ghai S. Teledentistry during COVID-19 pandemic. Diabetes Metab Syndr. 2020 Sep-Oct;14(5):933-935. DOI: $10.1016 /$ j.dsx.2020.06.029

[7] Petruzzi M, De Benedittis M. WhatsApp: une plateforme de télémédecine pour faciliter la consultation de médecine bucco-dentaire à distance et améliorer les examens cliniques. Oral Surg Oral Med Oral Pathol Oral Radiol. 2016 Mar; 121 (3): 248-54 DOI: $\underline{10.1016 / \text { j.oooo.2015.11.005 }}$

[8] Telles-Araujo GT, Caminha RDG, Kallás MS, Santos PSDS. Teledentistry support in COVID-19 oral care. Clinics (Sao Paulo). 2020 Jun 12; 75:e2030. DOI: 10.6061/clinics/2020/e2030

[9] Jain A, Grover V, Singh C, Sharma A, Das DK, Singh P, et al. Chlorhexidine: An effective anticovid mouth rinse. J among dental surgeons to adapt to the new scenario. Many preventive methods will be maintained after the end of the COVID-19 crisis. FFP2 masks for aerosol-generating procedures, hand disinfection, room ventilation, and face shields will continue to be used. New protocols have been recommended to reduce aerosol-generating operations: the concept of SAFE (Safe Aerosol-Free Emergent) dentistry may be an optimal compromise to ensure dental care during the highest peaks of virus waves due to the sudden variants of COVID-19. $(23,24)$

\section{CONCLUSION}

The overall goal of these recommendations is to protect dentists from COVID-19 while continuing to provide care, especially since several infectious variants of SARS-CoV2 are already present.

\section{CONFLICT OF INTEREST}

Authors have no conflicts of interest to declare.

Indian Soc Periodontol. 2021 Jan-Feb; 25(1):86-88. Doi: $10.4103 /$ jisp.jisp_824_20

[10] Vergara-Buenaventura A, Castro-Ruiz C. Use of mouthwashes against COVID-19 in dentistry. Br J Oral MaxillofacSurg. $2020 \quad$ Oct; $58(8): 924-927$. DOI: 10.1016/j.bjoms.2020.08.016

[11] Bidra AS, Pelletier JS, Westover JB, Frank S, Brown SM, Tessema B. Comparison of In Vitro Inactivation of SARS CoV-2 with Hydrogen Peroxide and Povidone-Iodine Oral Antiseptic Rinses. J Prosthodont. 2020 Aug; 29(7):599603. DOI: $10.1111 /$ jopr. 13220

[12] Lotfinejad N, Peters A, Pittet D. 2020. Hand hygiene and the novel coronavirus pandemic: the role of healthcare workers. J Hosp Infect. 2020 Aug; 105(4):776-777. DOI: $\underline{10.1016 / j . j h i n .2020 .03 .017}$

[13] Izzetti R, Nisi M, Gabriele M, Graziani F. COVID-19 Transmission in Dental Practice: Brief Review of Preventive Measures in Italy. J Dent Res. 2020 Aug; 99(9):1030-1038. DOI: 10.1177/0022034520920580

[14] Jamal M, Shah M, Almarzooqi SH, Aber H, Khawaja S, El Abed R, et al. Overview of transnational recommendations for COVID-19 transmission control in dental care settings. 2021 Apr; 27 Suppl 3: 655-644. DOI: 10.1111/odi.13431

[15] Dar-Odeh N, Elsayed S, Babkair H, Abu-Hammad S, Althagafi N, Bahabri R, et al. What the dental practitioner needs to know about pharmaco-therapeutic modalities of COVID-19 treatment: A review. J Dent Sci. 2021 Jul; 16(3): 806-816. DOI: 10.1016/J.JDS.2020.11.007

[16] Derruau S, Bouchet J, Nassif A, Baudet A, Yasukawa K, Lorimier S, et al. COVID-19 and Dentistry in 72 Questions: An Overview of the Literature. J Clin Med. 2021 Feb 16; 10(4):779. DOI: $10.3390 /$ jcm 10040779

[17] Mian M, Teoh L, Hopcraft M. Trends in Dental Medication Prescribing in Australia during the COVID-19 Pandemic. JDR Clin Trans Res. 2021 Apr; 6(2): 145-152. DOI: $10.1177 / 2380084420986766$

[18] Le Bon SD, Konopnicki D, Pisarski N, Prunier L, Lechien JR, Horoi M. Efficacy and safety of oral corticosteroids and olfactory training in the management of COVID-19-related loss of smell. Eur Arch Otorhinolaryngol. 2021 Aug; 278(8):3113-3117. DOI: 10.1007/s00405-020-06520-8 
[19] Zimmermann M, Nkenke E. Approaches to the management of patients in oral and maxillofacial surgery during COVID-19 pandemic. J Craniomaxillofac Surg. 2020 May;48(5):521-526.

DOI: 10.1016/J.JCMS.2020.03.011

[20] Passarelli PC, Rella E, Manicone PF, Garcia-Godoy F, D'Addona A. The impact of the COVID-19 infection in dentistry. Exp Biol Med (Maywood). 2020 Jun; 245(11):940-944. DOI: $10.1177 / 1535370220928905$

[21] Odeh ND, Babkair H, Abu-Hammad S, Borzangy S, AbuHammad A, Abu-Hammad O. COVID-19: Present and Future Challenges for Dental Practice. Int J Environ Res Public Health. 2020 Apr 30; 17(9):3151.

DOI: $10.3390 /$ ijerph17093151
[22] Salgarello S, Salvadori M, Mazzoleni F, Francinelli J, Bertoletti P, Audino E, et al. The New Normalcy in Dentistry after the COVID-19 Pandemic: An Italian CrossSectional Survey. Dent J (Basel). 2021 Jul 31; 9(8):86. DOI: $10.3390 / \mathrm{dj} 9080086$

[23] Beltrán-Aguilar E. Benzian H, Niederman R. Rational Perspectives on Risk and Certainty for Dentistry during the COVID-19 Pandemic. Am J Infect Control. 2021 Jan; 49(1): 131-133. DOI: $10.1016 /$ j.ajic.2020.06.007

[24] Arif TB. The 501.V2 and B.1.1.7 Variants of Coronavirus Disease 2019 (COVID-19): A New Time-Bomb in the Making? Infect Control Hosp Epidemiol. 2021 Jan 11; 12. DOI: $10.1017 /$ ice.2020.1434 